Study of intrinsic l ocal i zed vi brat i onal modes i $n$ mi cromechani cal oscill at or ar rays

\begin{tabular}{|l|l|}
\hline 著者 & $\begin{array}{l}\text { Sat o Nasayuki, Hubbar d B. E. , Engl i sh L. Q. , } \\
\text { Si ever s A. J., I I i c B. , Czapl ewski D. A. , } \\
\text { Crai ghead H. G }\end{array}$ \\
\hline $\begin{array}{l}\text { j our nal or } \\
\text { publ i cat i on t i tl e }\end{array}$ & Chaos \\
\hline vol une & 13 \\
\hline nunber & 2 \\
\hline page r ange & $702-715$ \\
\hline year & $2003-06-01$ \\
\hline URL & ht t p: //hdl . handl e. net $/ 2297 / 3822$ \\
\hline
\end{tabular}




\title{
Study of intrinsic localized vibrational modes in micromechanical oscillator arrays
}

M. Sato, B. E. Hubbard, L. Q. English, and A. J. Sievers

Laboratory of Atomic and Solid State Physics, Cornell University, Ithaca, New York 14853-2501

B. Ilic

Cornell Nanofabrication Facility and Department of Applied and Engineering Physics, Cornell University, Ithaca, New York 14853-5403

D. A. Czaplewski and H. G. Craighead

Department of Applied and Engineering Physics, Cornell University, Ithaca, New York 14853-3501

(Received 12 September 2002; accepted 21 November 2002; published 22 May 2003)

\begin{abstract}
Intrinsic localized modes (ILMs) have been observed in micromechanical cantilever arrays, and their creation, locking, interaction, and relaxation dynamics in the presence of a driver have been studied. The micromechanical array is fabricated in a $300 \mathrm{~nm}$ thick silicon-nitride film on a silicon substrate, and consists of up to 248 cantilevers of two alternating lengths. To observe the ILMs in this experimental system a line-shaped laser beam is focused on the 1D cantilever array, and the reflected beam is captured with a fast charge coupled device camera. The array is driven near its highest frequency mode with a piezoelectric transducer. Numerical simulations of the nonlinear Klein-Gordon lattice have been carried out to assist with the detailed interpretation of the experimental results. These include pinning and locking of the ILMs when the driver is on, collisions between ILMs, low frequency excitation modes of the locked ILMs and their relaxation behavior after the driver is turned off. () 2003 American Institute of Physics.
\end{abstract}

[DOI: $10.1063 / 1.1540771]$

An advance of the theory of nonlinear excitations in discrete lattices was the discovery that some localized vibrations in perfectly periodic but nonintegrable lattices could be stabilized by lattice discreteness. The modulational instability of extended large amplitude vibrational modes has been proposed as a mechanism for the realization of dynamical localization on the scale of the lattice constant. Although theoretically a variety of methods to excite the instability of a homogeneous vibrational mode have been proposed, these ideas have yet to be tested experimentally. Since the observation of nanoscale localized vibrational modes still cannot be achieved there is definite advantage to examining a macroscopic array, which is small enough so that the entire time dependence of the instability dynamics occurs in a practical measurement interval. This has been accomplished by using micromechanical silicon technology to fabricate up to $\mathbf{2 4 8}$ identical cantilevers with a 40 micron lattice constant. Optical techniques have been used to track the motion of individual cantilevers in the presence of an inertial driver. In addition to experimentally characterizing the modulational instability and identifying the best method for producing intrinsic localized modes a new discovery is the locking of the local mode amplitude with the driver frequency. Numerical simulations have been used to better understand the nature of this synchronization effect.

\section{INTRODUCTION}

The concept of nonlinear energy localization in periodic lattices characterizes a new class of dynamical excitations, namely, intrinsic localized modes (ILMs). ${ }^{1}$ In addition to theoretical and numerical studies involving nonlinear crystal dynamics ${ }^{2-7}$ applications to other topics have appeared such as magnetic systems, ${ }^{8-17}$ electron-phonon systems, ${ }^{18,19}$ reaction dynamics, ${ }^{20}$ molecular biophysics, ${ }^{21,22}$ and latticeassisted energy/charge transfer in polarizable matter. ${ }^{23}$ Some of these efforts devoted to examining the nonlinear dynamics of nanoscale lattices have made contact with other possible applications for ILMs such as in friction ${ }^{24}$ and crack propagation. ${ }^{25}$ Still other larger scale applications deal with ILMs in Josephson arrays, ${ }^{26,27}$ E\&M ILMs in optical switches ${ }^{28}$ and in nonlinear photonic crystal wave guides. ${ }^{29,30}$ The largest scale application has to do with localized multibunch modes in accelerators. ${ }^{31}$ Thus from condensed matter physics to arrays used in high technology, one sees a new class of problems emerging, which share a common denominator.

At the smallest scale, details of ILM quantization ${ }^{18,32,33}$ are still to be explored. For a classical nonlinear oscillator array, there are a number of characteristic ILM properties, probed theoretically, such as their interaction with an ac driver, ${ }^{14,34-36}$ their propagation ${ }^{5,37-40}$ and amplitude dependent mobility ${ }^{4,6,40-42}$ in a discrete lattice potential, ${ }^{43,44}$ as well as their interactions with impurities, ${ }^{45-50}$ that still need 
to be examined experimentally. Note that strongly excited $\mathrm{ILMs}^{42}$ can be trapped anywhere in the lattice, so they also could approach impurity mode behavior. Thus the exploration of the amplitude-dependent properties of ILMs is one of the important experimental issues at the present time.

Although some experimental studies have been reported for large scale mechanical systems, ${ }^{51,52}$ for somewhat smaller Josephson-junction arrays, ${ }^{26,27,53,54}$ and for nanoscale lattices, ${ }^{15-17,55}$ none of these studies have examined the driven amplitude dependent trapping phenomena. The low resonant frequency of the mechanical systems makes the time scale too long to perform the necessary experiments. ILMs can be seeded in Josephson-junction arrays and they are stable so that trapped ILMs can be measured; however, the high oscillation frequency, $1 \mathrm{GHz}$ to $10 \mathrm{THz},{ }^{56}$ makes it difficult to observe their dynamical motion. Finally, observing the dynamics in nanoscale lattices has not yet been accomplished. Recently micro-electro-mechanical system (MEMS) silicon technology has matured sufficiently so that now it is relatively straight forward to make many identical elements. ${ }^{57-59}$ While previous experimental studies of individual micromechanical oscillators have focused on issues of nonlinearity and specific applications, ${ }^{60-63}$ in this paper we describe our experimental investigation of ILM creation, relaxation, locking, and interaction in 1D coupled oscillator arrays of the cantilever design.

In the next section the experimental and numericalsimulation procedures are described. An optical arrangement with a 1D charge coupled device (CCD) is used to display the motion of localized modes. Numerical simulations have been made using coupled nonlinear Klein-Gordon equations to represent the oscillator array. In Sec. III, the experimental results are presented and compared with the simulations. The breakup of the uniform mode excitation, the development of localized modes, and stationary localized modes locked to the driver frequency have been observed in this time dependent investigation. Simulations play an important role as they are used to interpret and understand these experimental observations. The conclusions are presented in Sec. IV.

\section{EXPERIMENTAL DETAILS}

\section{A. Physical}

\section{Fabrication and linear measurements to characterize cantilever coupling}

To fabricate $\mathrm{SiN}_{x}$ cantilever arrays on a silicon substrate, the starting film is a low stress silicon nitride layer, silicon rich to alleviate tensile stresses. After coating with a photoresist mask it is exposed and then etched via a $\mathrm{CF}_{4}$ plasma in a reactive ion chamber. Next, the silicon substrate is undercut using an anisotropic $\mathrm{KOH}$ etch, thus releasing the $\mathrm{SiN}_{x}$ cantilevers. A top view of the resulting structure is shown in Fig. 1(a). The relative scale of the cantilevers and overhang can be seen. The overhang region provides the coupling between the cantilevers. A 3D rendition of one unit cell of the resulting array is shown in Fig. 1(b). The physical characteristics of the four oscillator arrays studied here are given in Table I.

To examine the coupling properties of the overhang between the cantilevers, some initial tests have been carried out

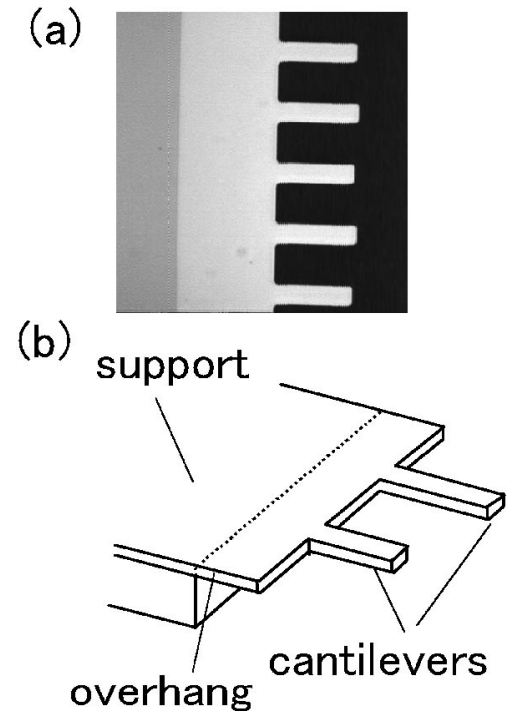

FIG. 1. Characteristic dimensions of a di-element type silicon nitride cantilever array. (a) Top view photograph. White region to the right is the $300 \mathrm{~nm}$ thick $\mathrm{SiN}_{x}$ array and overhang, while the dark gray region on the left is the film supported by the substrate. The parameters for this sample (D) are given in Table I. (b) 3D sketch showing one unit cell.

with small arrays composed of nine identical cantilevers. These are samples A and B in Table I. A piezoelectric transducer (PZT) with variable frequency is used to drive the monocantilever array to obtain the frequency dependent response of individual cantilevers.

The optical apparatus used to measure the dependence of the spectral bandwidth on the overhang is shown in Fig. 2. The sample is attached to the PZT and situated in an evacuated chamber maintained below 1 mTorr at ambient temperature. A cantilever is chosen, and a laser beam focused on it, as can be seen in the figure. A variable frequency ac voltage drives the PZT which uniformly shakes the entire sample so that the cantilever array experiences a common acceleration. The resulting deflection of the laser beam from the oscillating cantilever is measured by a position sensitive photodiode detector (PSD), with output voltage proportional to the optical spot position. For a linear response of the array the PZT ac voltage is typically set to $0.05 \mathrm{~V}$; for examining the nonlinear shift of a resonance frequency, and hence the anharmonicity of the system, the driving voltage is $\sim 0.1 \mathrm{~V}$. For ILM creation it is $\sim 10 \mathrm{~V}$.

Figures 3(a) and 3(b) show the signal versus driving frequency for test samples A and B, respectively. These two samples, which are identical except for different overhang widths, produce different spectral bandwidths as expected. Figures 3(a) and 3(b) clearly show that the bandwidth increases with the length of the overhang. Ignoring for the moment any normal modes of the overhang itself, the cantilevers should produce nine degrees of vibrational freedom. The nine peaks observed are the resonant frequencies of these nine normal modes. The solid and dotted curves denote the spectra taken of the center and edge cantilever, respectively, so that all modes are counted.

With the coupling between oscillators characterized, the design of the large oscillator arrays can now be considered. It 
TABLE I. Summary of the characteristics of the micromechanical samples.

\begin{tabular}{lrrrr}
\hline \hline Sample label & \multicolumn{1}{c}{ A } & \multicolumn{2}{c}{ B } & C \\
\hline Type & Mono-element & Mono-element & Di-element & Di-element \\
Thickness $(\mathrm{nm})$ & 300 & 300 & 300 & 300 \\
Pitch $(\mu \mathrm{m})$ & 110 & 110 & 65 & 40 \\
Length $(\mu \mathrm{m})$ & 50 & 50 & $50 / 55$ & $50 / 55$ \\
Width $(\mu \mathrm{m})$ & 10 & 10 & 15 & 15 \\
Total number & 9 & 9 & 152 & 248 \\
Overhang $(\mu \mathrm{m})$ & 28.5 & 35.6 & 70 & 67 \\
$f_{a}(\mathrm{kHz})^{\mathrm{a}}$ & 180.43 & 186.2 & 136.1 & 147.0 \\
$f_{b}(\mathrm{kHz})^{\mathrm{a}}$ & & & 133.0 & 143.2 \\
$f_{c}(\mathrm{kHz})^{\mathrm{a}}$ & & & 121.2 & 127.7 \\
$f_{d}(\mathrm{kHz})^{\mathrm{a}}$ & 171.43 & 158.4 & 72.7 & 60.8 \\
\hline \hline
\end{tabular}

${ }^{a}$ Resonant frequencies at the upper zone center, upper zone boundary, lower zone center, and lower zone boundary, as shown in Fig. 7. For the mono-element type arrays, only two frequencies are specified which correspond to the same modes in folded dispersion curve as if the samples were of the di-element type.

is known that cantilevers of this design have a hard nonlinearity. ${ }^{61,62}$ The procedure of choice for the production of ILMs is to drive a uniform mode to large amplitude so that it becomes unstable and breaks up into localized excitations. To achieve the large amplitude uniform mode instability for an array with hard anharmonicity, the highest frequency uniform mode of the array needs to be driven. ${ }^{64}$

For a mono-type cantilever 1D lattice this would be the zone-boundary excitation. (Although our arrays have fixed boundary conditions they are sufficiently large that terminology consistent with periodic boundary conditions will be used throughout the paper.) To excite the zone boundary mode shown in Fig. 4(a) would require a special driver, one that could produce $\pi$ out-of-phase amplitude on neighboring cantilevers. In order to use the PZT driver, which accelerates the entire lattice uniformly, two different-length cantilevers per unit cell have been constructed in arrays $\mathrm{C}$ and $\mathrm{D}$, as displayed in Fig. 1. With this di-element array, the dispersion curve is folded over [see Fig. 4(b)] so that the highest frequency vibrational mode is now at the zone center. This optic-like mode can be excited with the PZT driven at the appropriate frequency. Any ILMs should appear near the top of the upper band as shown in the figure.

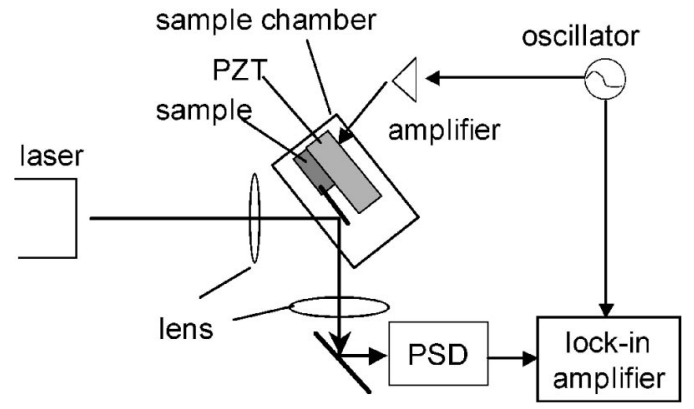

FIG. 2. Experimental setup for a one-cantilever linear response measurement. The cantilever array is in a vacuum chamber. A PZT is employed to drive the sample. A beam from a $\mathrm{He}-\mathrm{Ne}$ laser is focused on one cantilever. A position-sensitive photodiode detector (PSD) which outputs voltage proportional to the laser beam position is used to pick up the deflection of the reflected laser beam. By scanning the frequency of the oscillator and measuring the ac voltage with the lock-in amplifier, linear spectra are obtained.

\section{Linear measurements of large di-element cantilever arrays}

The frequency dependent linear response of individual cantilevers for a large di-element array (sample D) is shown in Fig. 5. The laser beam, shown in Fig. 2, is now focused on a cantilever, near the center of the sample. The drive frequency is incremented in $100 \mathrm{~Hz}$ steps in the lower frequency region and $50 \mathrm{~Hz}$ steps in the higher frequency region. For each frequency point, a measurement takes about three seconds. Optic-like normal modes can be activated by the uniform driver, and can be seen in this figure. As expected, there are two pass bands, with the upper band $(143.2-147.0 \mathrm{kHz})$ much narrower than the lower one $(60.8-127.7 \mathrm{kHz})$. Due to the coupling of the driver to the normal modes the frequency dependence of the response decreases with increasing frequency in the lower band, and increases with increasing frequency in the upper band. There is at least one other band at around $235-240 \mathrm{kHz}$, which may be related to the vibration of the overhang. However,

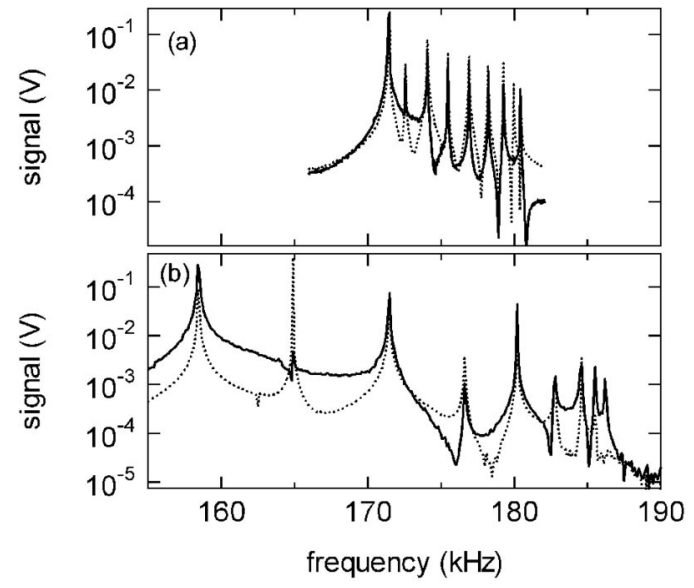

FIG. 3. Linear spectra for mono-element arrays showing the dependence of the bandwidth on the overhang. (a) Sample A with $28.5 \mu \mathrm{m}$ overhang, and (b) sample B with $35.6 \mu \mathrm{m}$ overhang. Samples A and B contain nine cantilevers; see Table I for more details. Solid and dotted curves correspond to the spectrum for the center and for the edge cantilever, respectively. 

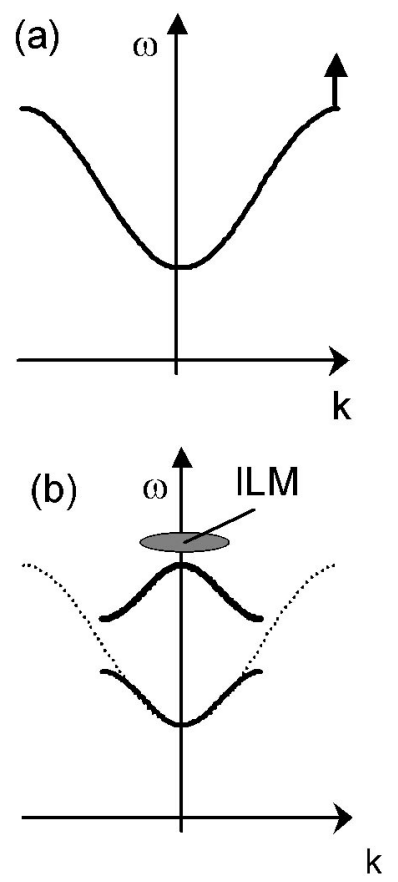

FIG. 4. Schematic dispersion curves. (a) Mono-element cantilever array: an ILM can be expected to appear at the zone boundary frequency for hard nonlinearity. (b) Di-element cantilever array: the dispersion curve for the mono-element array (thin dotted line) is folded back and a stop band appears. For hard anharmonicity ILMs will be created at the zone center.

this region is beyond the frequency limit of the photosensitve detector and the lock-in amplifier, and it has not been measured accurately.

To examine the response of the system to large amplitude excitation and in particular to explore ILM dynamics, it is necessary to measure a large number of cantilevers simultaneously. Figure 6 shows the experimental setup for measuring ILM dynamics versus time. In Fig. 6(a) the $\mathrm{He}-\mathrm{Ne}$ laser beam is focused with a cylindrical lens into a line along the static array. The reflected beam is then imaged onto a $1 \mathrm{D}$ CCD camera. The PZT is driven with a voltage-controlled oscillator for variable or constant frequency operation as shown in the figure. The switch and the ramp generator are synchronized with the camera by using a pulse generator. Images are captured by a computer, which is triggered by the pulse generator. The speed of the camera, about $18 \mathrm{kHz}$, is insufficient to monitor the sinusoidal motion of the cantilevers. However, as the vibration of a cantilever grows, the reflected laser beam increasingly misses the 1D CCD camera as illustrated in Fig. 6(b), and the image of that particular cantilever becomes darker.

The maximum lateral capturing speed of the camera can be defined as the array pitch divided by the camera period, i.e., $18 \times 10^{3} \mathrm{a} / \mathrm{s}$, where $a$ is the cantilever pitch. This is sufficient to observe the fastest traveling ILMs, which move at speeds of up to $14 \times 10^{3} \mathrm{a} / \mathrm{s}$. It should be emphasized that ILMs excited near the top of the band are far slower than this maximum speed. Consequently, the capturing speed of the camera is fast enough to observe both the lateral motion of an ILM and its time development. This relatively straightforward observational method permits a systematic monitoring

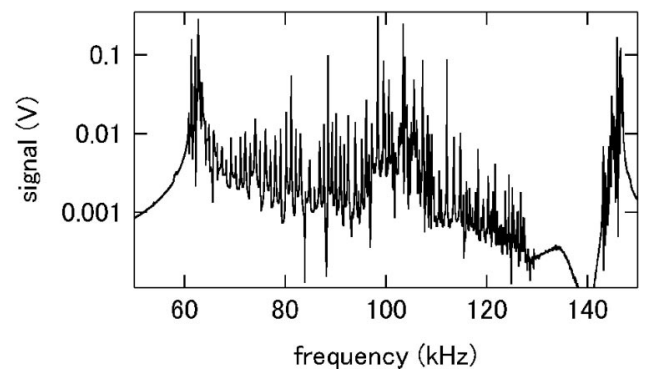

FIG. 5. Linear spectrum obtained for a large di-element cantilever array. The laser was focused on a cantilever near the middle of sample D that contains 248 cantilevers. Frequency is step incremented in $100 \mathrm{~Hz}$ steps over the lower frequency region and in $50 \mathrm{~Hz}$ steps in the higher frequency region. The lower band begins at $60.8 \mathrm{kHz}$ and ends at $127.7 \mathrm{kHz}$. The upper band begins at $143.2 \mathrm{kHz}$ and ends at $147.0 \mathrm{kHz}$.

of the excitation pattern for large amplitude ILM creation and interaction with a driver.

It has been shown theoretically that changing the frequency (chirping) of a large amplitude driver is an effective method by which to excite a nonlinear system to large amplitude so that the resulting instability produces ILMs. ${ }^{35,65}$ Since the resonant frequency of the nonlinear cantilevers in-

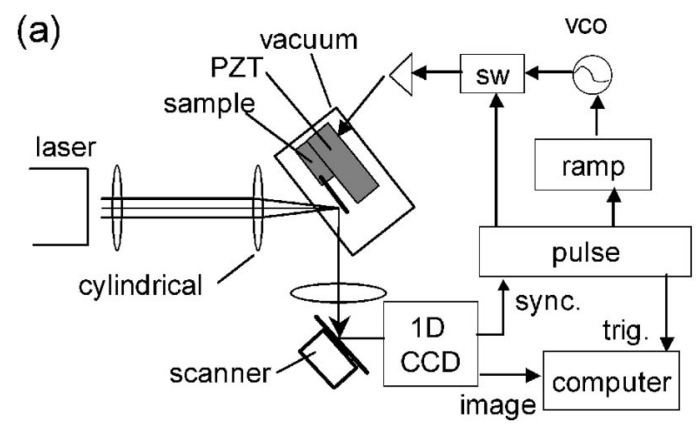

(b)

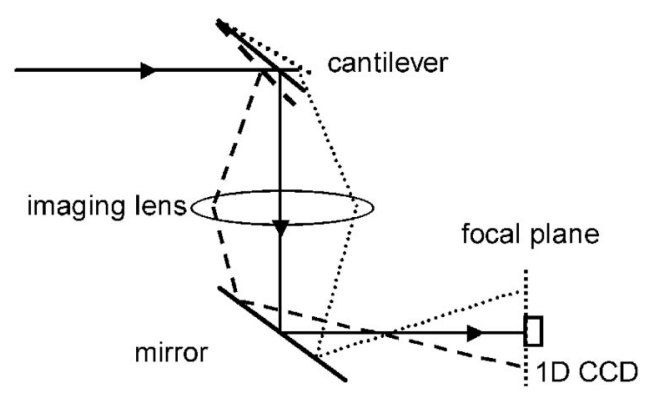

FIG. 6. (a) Experimental setup for the ILM measurements. A beam from a $\mathrm{He}-\mathrm{Ne}$ laser is focused along the array by using a cylindrical lens. The elliptical spot from the cylindrical lens is positioned so that the short axis of the ellipse lies along the length of the cantilever, while the longer axis encompasses many cantilevers. A 1D CCD is used to detect the beam reflected from the cantilevers. A voltage controlled oscillator (vco), switch (sw) and amplifier are used to drive the PZT. The frequency of the vco is controlled by a ramp generator. A pulse generator, which controls the switch, and the ramp generator are synchronized to the camera. A scanner attached to the mirror is driven by an oscillator (not shown in this figure). Twodimensional images can be captured by synchronized scanning of the mirror and the camera. This feature is used to observe the amplitude pattern of stationary ILMs. (b) Schematic showing the relation between cantilever amplitude and detector response. At large vibration amplitudes the deflection of the laser beam from the cantilever changes and the image at the CCD site becomes darker. In this picture the elements of the 1D CCD camera are arranged horizontally. 
creases with amplitude, it is necessary to increase the driver frequency with time to track the highest resonant frequency. In addition, since the oscillators are necessarily damped, $\mathrm{cw}$ driving is required for steady state observation of the resultant ILMs.

A scanner attached behind the mirror in front of the CCD as shown in Fig. 6(a) can be used to obtain a twodimensional image of the cantilevers. This oscillator driven scanner is synchronized with the camera. During the time development measurement of ILMs, which is the main focus of most experiments, this mirror is mechanically fixed to observe individual cantilever motion.

\section{B. Numerical}

The lowest frequency $\omega$ of a simple cantilever with one end fixed is given by ${ }^{66}$

$$
\omega^{2}=\frac{(3.52)^{2}}{L^{4}}\left(\frac{E I}{\rho t w}\right)=\frac{(3.52)^{2}}{12 L^{3}} \frac{E}{m} t^{3} w=\frac{k}{m} .
$$

Here $E$ is the Young's modulus, the second moment-of-area $I=t^{3} w / 12$, where $t$ is the thickness of the beam, and $w$ is its width (see Table I for these values), $L$ is the length of the beam, and $\rho$ is the density of the material.

Our experimental system consists of an array of cantilever beams that are coupled together by the overhang region between them. Since the two cantilevers in a unit cell have different lengths, then, according to Eq. (1), both their linear spring constants and masses will be different. As a first approximation, the dynamics of the overhang will be ignored, so that it acts only as a massless coupler between cantilevers. For small oscillations an individual cantilever in the array is assumed to obey Eq. (1) but with an effective spring and mass, in order to include some contribution of the overhang. For the large amplitude problem, each cantilever is then represented by a mass and an onsite potential which has both harmonic (quadratic) and anharmonic (hard quartic) terms. The coupling between cantilevers is assumed to be harmonic for all cantilever amplitudes. Such a di-element lattice model obeys the nonlinear Klein-Gordon equation, ${ }^{67}$ namely,

$$
\begin{gathered}
m_{a} \frac{d^{2}}{d t^{2}} x_{a i}+\frac{m_{a}}{\tau} \frac{d}{d t} x_{a i}+k_{2 a} x_{a i}+k_{4 a} x_{a i}^{3} \\
+k_{I}\left(2 x_{a i}-x_{b i}-x_{b i-1}\right)=m_{a} \alpha, \\
m_{b} \frac{d^{2}}{d t^{2}} x_{b i}+\frac{m_{b}}{\tau} \frac{d}{d t} x_{b i}+k_{2 b} x_{b i}+k_{4 b} x_{b i}^{3} \\
+k_{I}\left(2 x_{b i}-x_{a i+1}-x_{a i}\right)=m_{b} \alpha,
\end{gathered}
$$

where the subscripts $a$ and $b$ correspond to the different length cantilevers, $x_{a i}$ and $x_{b i}$ are the displacements of the cantilever ends, $m_{a}$ and $m_{b}$ are their masses, $\tau$ is a linear (energy) lifetime, $k_{2 a}$ and $k_{2 b}$ are the onsite harmonic spring constants, $k_{4 a}$ and $k_{4 b}$ are onsite quartic spring constants, and $k_{I}$ is the harmonic coupling constant. The PZT does not apply a force directly to the cantilevers, but causes acceleration and deceleration of the cantilever's inertial frame with magnitude $\alpha$.
The masses are estimated from the density of silicon nitride and the size of the cantilevers. The lifetime $\tau$ is experimentally determined. In order to determine the spring constants $k_{2 a}, k_{2 b}$, and $k_{I}$ experimentally, four frequencies have been measured: the maximum and minimum frequencies for the upper and for the lower bands. These points on a dispersion curve are identified in Table I.

By choosing the top three experimental frequencies for the fit to the small oscillation case, a good approximation to the upper branch of the dispersion curve can be obtained. The lowest experimental frequency (lower branch, zone center) is not reproduced within our model. One way to obtain different bandwidths is to introduce longer-range interactions, which would stem from the dynamical properties of the overhang. To reproduce the entire experimental dispersion curve takes up to sixth nearest-neighbor interactions. Since ILMs are created at the top of the upper branch, the exact shape of the lower branch is not expected to play a crucial role so this long-range interaction is not included in our simulations.

With the harmonic spring constants of the model chosen, its linear response can be determined and the dispersion curve obtained. The system is driven with a uniform distribution of acceleration noise, with $\alpha$ ranging between $\pm 10^{-3} \mathrm{~m} / \mathrm{s}^{2}$ (compared to the $\alpha \sim 10^{+4} \mathrm{~m} / \mathrm{s}^{2}$ that will be needed for ILM production). The beam displacements of the entire array are recorded as a function of time. A Fourier transform (FT) of a single oscillator displacement as a function of time yields the frequencies that the particular cantilever experiences during the given time interval. Likewise, a FT (taken over a finite spatial interval) of the displacements of all the cantilevers at any instant of time will yield the excited wave vector modes that are participating in the collective motion. Performing FTs in both space and time changes the real-space data $(x, t)$ representation to reciprocal space $(\omega, k)$. Such a two-dimensional FT of the beam displacement data is shown in Fig. 7(a). The time interval is equivalent to 1000 periods of oscillation. A one-dimensional FT for the oscillations of a single cantilever from the middle of the sample is shown in Fig. 7(b). In both of these frames it is clear that the simulated bandwidth is almost the same for the acoustic and optic branches.

The nonlinear onsite quartic spring constant in Eqs. (2) and (3) is determined in the following manner: (1) $k_{4 a}$ and $k_{4 b}$ are set equal to each other and (2) the value is determined so that the upper-branch frequency in a simulation shows the same frequency shift as for experiment at a power level below the threshold for the uniform mode instability. This power still needs to be sufficiently high so that the nonlinear frequency shift of the resonant frequency can be determined accurately. A complete list of simulation parameter values can be found in Table II.

\section{RESULTS AND DISCUSSION}

\section{A. Trapping and locking}

\section{Experiment}

Two sequential time-dependent response measurements of the 152-cantilever array (sample C) for a large amplitude 

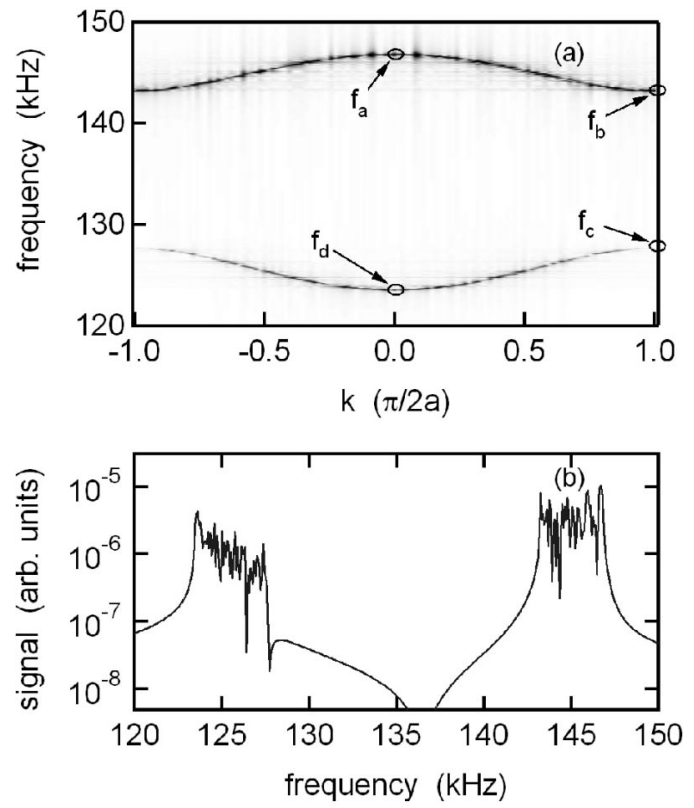

FIG. 7. Simulated linear response for a di-element cantilever array. The physical parameters of the di-element array are given in Table II. Applying low noise amounting to $\alpha=0 \pm 10^{-3} \mathrm{~m} / \mathrm{s}^{2}$, the displacements are measured as a function of time. (a) The time-space Fourier transform of the shorter cantilever displacements gives the excitation pattern in $k$ space only where normal modes exist. The three experimental frequencies, 147.0, 143.2, and $127.7 \mathrm{kHz}$ at the top and bottom of the upper band, and top of the lower band of sample D are reproduced. The bottom of the lower band frequency $(123.3 \mathrm{kHz})$ in simulation is much higher than the experimental one $(60.8$ $\mathrm{kHz}$ ). (b) The time Fourier spectrum of a single cantilever displacement pattern.

driver are shown in Fig. 8. The cantilever positions can be identified by the white lines to the left of the time $=0$ marker. The high-power, PZT driver $(\sim 20 \mathrm{~V})$ is chirped from the top of the optic band, $f_{a}$, to $1.011 f_{a}$ between time $=0$ and 14.2 $\mathrm{ms}$ as indicated by the dotted vertical line in the figure. The CCD camera images a portion of the sample, which is 100 cantilevers in this case. The dark tracks versus time identify large amplitude localized excitations. Although the starting conditions are the same for the experiments shown in Figs. 8 (a) and 8(b) different results are observed as would be expected for a process initiated by random noise. Note that some moving ILMs form during the chirp phase. At longer times between $14.2 \mathrm{~ms}$ and $72.7 \mathrm{~ms}$, with a cw driver some ILMs continue to receive energy while the others die out. At some point the large amplitude ILMs become trapped at a lattice site. This can be seen around lattice site 60 in Fig. 8(a) and at two locations, namely, 38 and 80 in Fig. 8(b). After the driver is turned off at time $=72.7 \mathrm{~ms}$ the trapped ILMs decay.

TABLE II. List of parameters used in the simulations.

\begin{tabular}{lll}
\hline \hline \multicolumn{1}{c}{ Parameters } & \multicolumn{1}{c}{ Cantilever $a$} & \multicolumn{1}{c}{ Cantilever $b$} \\
\hline Mass & $5.46 \times 10^{-13} \mathrm{~kg}$ & $4.96 \times 10^{-13} \mathrm{~kg}$ \\
Damping constant, $\tau$ & $8.75 \mathrm{~ms}$ & $8.75 \mathrm{~ms}$ \\
Harmonic, $k_{2}$ & $0.303 \mathrm{~kg} / \mathrm{s}^{2}$ & $0.353 \mathrm{~kg} / \mathrm{s}^{2}$ \\
Anharmonic, $k_{4}$ & $5.0 \times 10^{8} \mathrm{~kg} / \mathrm{s}^{2} \mathrm{~m}^{2}$ & $5.0 \times 10^{8} \mathrm{~kg} / \mathrm{s}^{2} \mathrm{~m}^{2}$ \\
Harmonic intersite, $k_{I}$ & $0.0241 \mathrm{~kg} / \mathrm{s}^{2}$ & $0.0241 \mathrm{~kg} / \mathrm{s}^{2}$ \\
\hline \hline
\end{tabular}

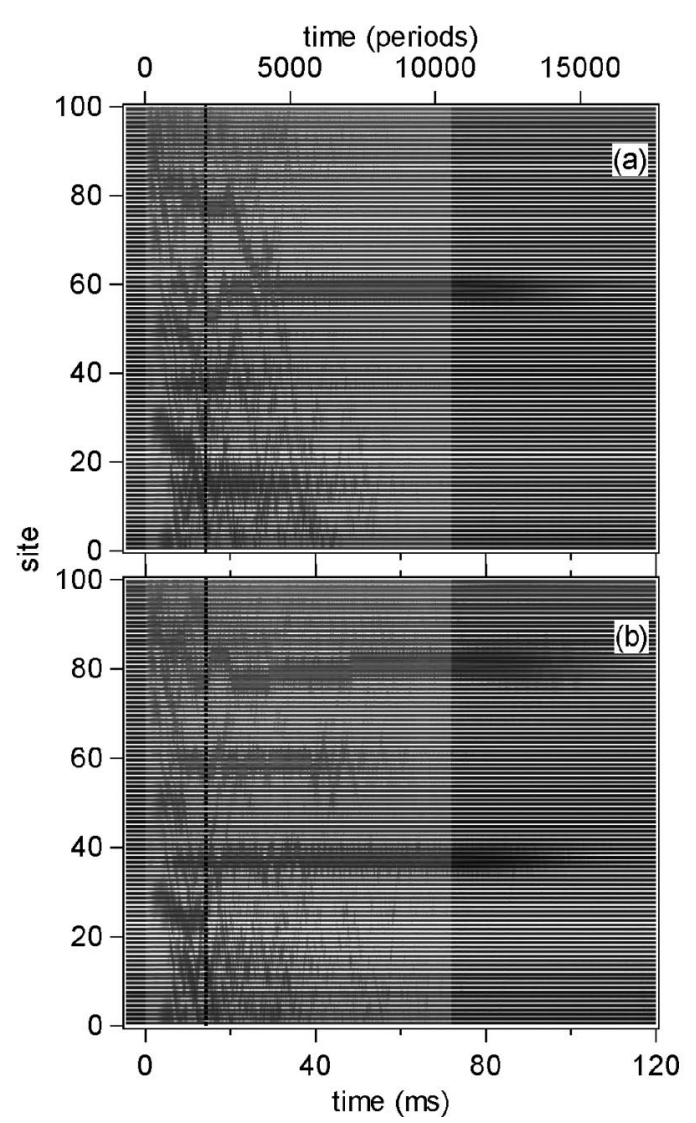

FIG. 8. Cantilever excitation versus time showing the production, interaction and decay of ILMs. These experimental results for sample $\mathrm{C}$ are taken with the 1D CCD camera. Frames (a) and (b) have identical starting conditions. The PZT frequency is chirped from $f_{a}=136.3 \mathrm{kHz}$ to $1.011 f_{a}$. The chirp ends at $14.2 \mathrm{~ms}$ (dotted line). The dark regions identify localized excitations. Highlighted region corresponds to the time where the pulse is on. Some localized excitations become trapped during this $\mathrm{cw}$ phase. Pulse duration is $72.7 \mathrm{~ms}$.

Figure 9 shows cantilever responses versus time with the high-power, chirped driver for the 248-element array, sample $\mathrm{D}$ in Table I. Before the pulse is turned on (time $<0$ ), each stripe corresponds to a stationary cantilever. The PZT voltage is again about $20 \mathrm{~V}$. The viewing size is now 230 cantilevers. Figures 9(a) and 9(b) show different results for identical starting conditions. Here, the driver frequency is chirped from $0.9986 f_{a}$ to $1.016 f_{a}$. Figure 9(c) shows the results for a slightly different starting condition, where the driver frequency is chirped from $0.9986 f_{a}$ to $1.034 f_{a}$. As long as the chirping starts near the top of the band and ends $2-3 \%$ higher than $f_{a}$, the different chirping schemes give similar results. The pulse duration is $48.9 \mathrm{~ms}$ and the chirp time is $16.2 \mathrm{~ms}$ (dotted line). The pulse interval is highlighted in these figures. At early times ILMs are observed to form, move, oscillate and hop until the chirp ends; then a few ILMs continue to pick up energy and become trapped at lattice sites. Using an optical probe coupled to a spectrum analyzer to monitor the center of such a pinned ILM confirms that the fixed mode is frequency-locked to the driver. After the pulse, these stationary ILMs lose energy, become broader, break free from the trapping site, and sometimes oscillate about it. Note that these oscillations range over 10-20 lattice sites and hence they have a much larger am- 


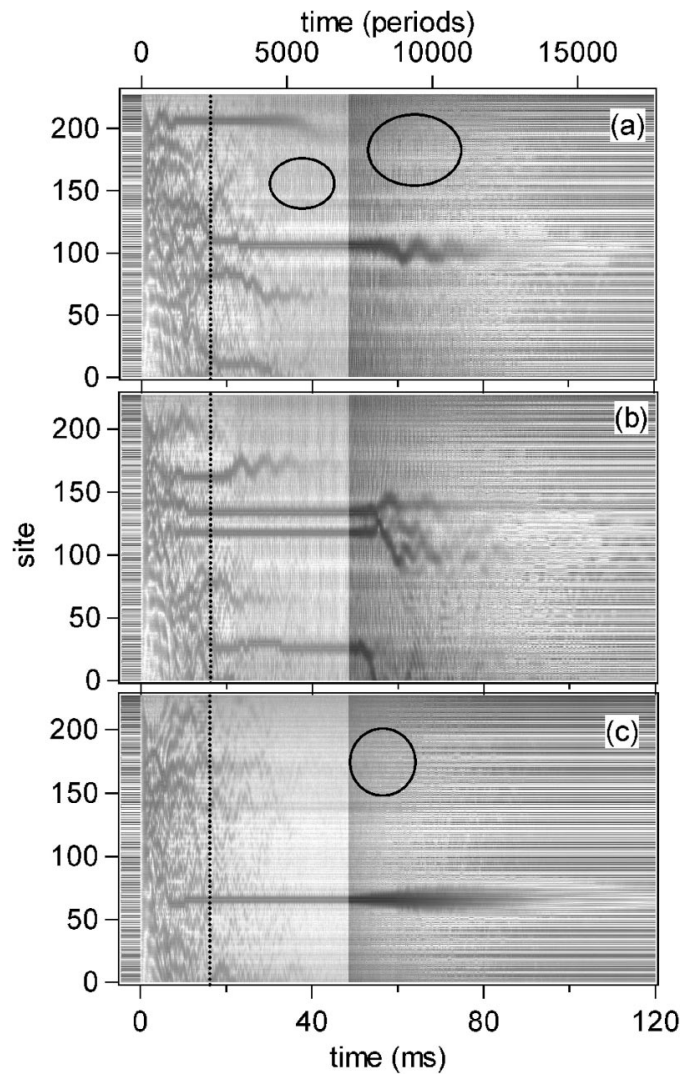

FIG. 9. Cantilever excitation versus time showing the production and decay of ILMs. Experimental results for sample D taken with the 1D CCD camera. Frames (a) and (b) have identical starting conditions. The frequency of the PZT is chirped from $0.9986 f_{a}$ to $1.016 f_{a}$. For frame (c) the chirp extends from $0.9986 f_{a}$ to $1.034 f_{a}$. Chirp ends at $16.2 \mathrm{~ms}$ (dotted line). Dark patterns identify localized excitations. The pulse is on over the highlighted time interval and its duration is $48.9 \mathrm{~ms}$. Ovals indicate some of the many regions where fairly uniform background excitations can be seen. The amplitude of this normal mode noise is larger in (a) and (b) than it is in (c). After Ref. 68.

plitude than the one unit cell oscillations described in Refs. 38 and 43. The moving ILMs have speeds ranging from 2-6 lattice sites per millisecond. This should be compared to the maximum possible speed of an optic branch linear mode wave packet of about 13 lattice sites per millisecond. The oval identifiers in Figs. 9(a) and 9(c) indicate regions where fairly uniform background excitations can be seen. Such patterns exist even after the pulse. The amplitude of such excitations in Fig. 9(c) is less than in frames (a) and (b) and probably accounts for the absence of any motion of the trapped ILM after the pulse is turned off. A brief description of some of these results has already been given. ${ }^{68}$

Close inspection of the large-amplitude trapped ILMs in Fig. 9 reveals an experimental artifact in the central region of the ILM image. The experiments indicate a smaller amplitude at the center of the mode than actually exists there. A systematic investigation shows this feature is false. By taking a 2D image of two trapped ILMs, which are shown in Fig. 10 , it is possible to identify the single peaked nature of the excitation. The two arrows in the figure identify the center of the two localized excitations. In each case the large amplitude peak is centered on the short cantilever.

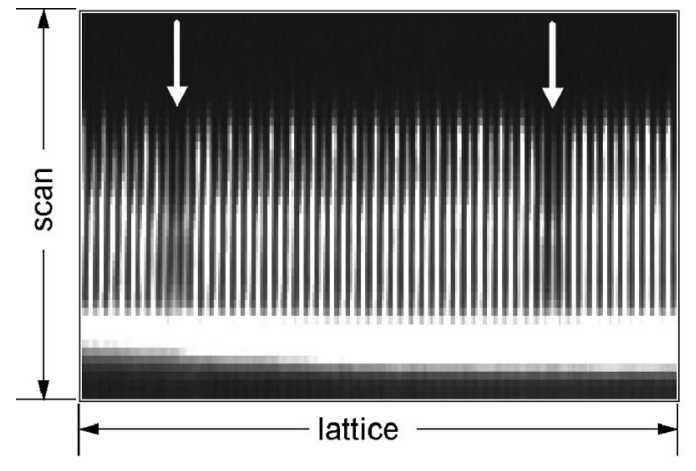

FIG. 10. Two-dimensional image of the amplitude pattern for sample D showing two trapped ILMs. Note that each ILM is centered on a short cantilever site. The two stationary ILM patterns are obtained by scanning the mirror in front of the CCD shown in Fig. 6. A scanner, synchronized with the $\mathrm{CCD}$, is used to vibrate the mirror. The cantilevers and the overhang region are illuminated by the laser and the small amplitude ones appear bright in this image. Strongly vibrating cantilevers appear darker. The shape of each pinned ILM is single peaked.

\section{Simulations}

To understand in more detail a number of the features observed in Figs. 8 and 9 it is necessary to turn to numerical simulations, which have parameters very similar to those in the experiment. Figure 11 summarizes some of the results of such an investigation. The chirping scheme used in this simulation is shown in Fig. 11(a). The system is initialized with a small amount of random noise. The system is then allowed to settle for 500 periods of the resonant frequency, at which point the driver is turned on $($ time $=0)$. The driver frequency starts at the top of the upper band, then continuously increases linearly to frequency $1.027 f_{a}$ over a time interval of 2500 periods. When the uniform mode becomes highly excited the noise perturbation triggers the modulational instability. In the $\mathrm{cw}$ mode the fixed driver frequency is $1.027 f_{a}$ until the driver is turned off at 7500 periods.

Figure 11(b) shows a density-plot of the energy of each lattice site as a function of time for a typical simulation result. The energy of a particular lattice site in the di-element array, at a given instant of time, is

$$
\begin{aligned}
E_{a_{i}}= & \frac{1}{2} m_{a}\left(\frac{d}{d t} x_{a_{i}}\right)^{2}+\frac{1}{2} k_{2 a} x_{a_{i}}^{2}+\frac{1}{4} k_{4 a} x_{a_{i}}^{4} \\
& +\frac{1}{4} k_{I}\left(x_{a_{i}}-x_{b_{i}}\right)^{2}+\frac{1}{4} k_{I}\left(x_{a_{i}}-x_{b_{i-1}}\right)^{2}, \\
E_{b_{i}}= & \frac{1}{2} m_{b}\left(\frac{d}{d t} x_{b_{i}}\right)^{2}+\frac{1}{2} k_{2 b} x_{b_{i}}^{2}+\frac{1}{4} k_{4 b} x_{b_{i}}^{4} \\
& +\frac{1}{4} k_{I}\left(x_{b_{i}}-x_{a_{i+1}}\right)^{2}+\frac{1}{4} k_{I}\left(x_{b_{i}}-x_{a_{i}}\right)^{2},
\end{aligned}
$$

where $i$ indexes the unit cell number, and the actual lattice site number is given by $j=2 i-1$ and $j=2 i$ for the long (cantilever $a$ ) and short (cantilever $b$ ) sites, respectively. The energy consists of the kinetic energy and onsite potential terms, as well as the potential energy stored in the coupling springs. The parameter set used here for sample D is given in Table II. The total number of cantilevers is 250 with fixed- 


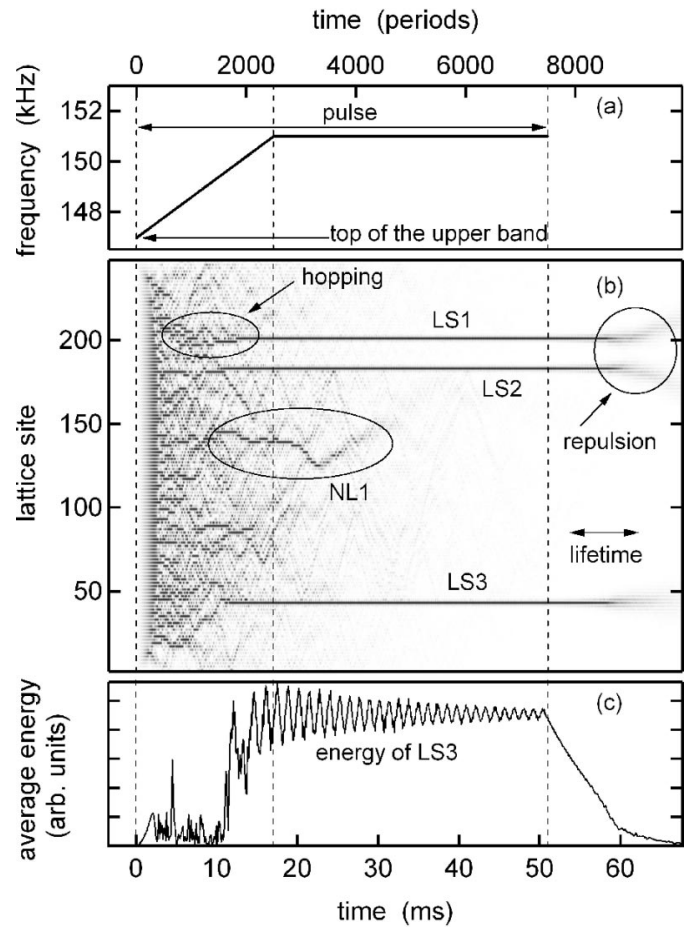

FIG. 11. Computer simulations of ILM production, dynamics, and decay. Cantilever parameters are listed in Table II. (a) Driver frequency as a function of time for this simulation. Pulse is on from 0 to 7500 periods. Driver frequency starts at $f_{a}$, increases up to $1.027 f_{a}$ linearly until 2500 periods, and then, remains at that frequency. The magnitude of the acceleration is kept constant, $\alpha=1.0 \times 10^{4} \mathrm{~m} / \mathrm{s}^{2}$. (b) A density plot of the energy versus time. Dark regions identify localized modes. During the time that the $\mathrm{cw}$ driver is on three locked stationary ILMs (LS1, LS2, LS3) are found. NL1 identifies an ILM, which is not locked to the driver. Hopping motion of ILMs can be seen in the oval markers. (c) Energy as a function of time for the ILM labeled LS3 averaged over three lattice sites. The characteristic oscillation frequency of the slowly decaying feature is $0.0056 f_{a}$ with a $Q$ $\sim 13$. Horizontal arrow shows a linear energy lifetime $(8.75 \mathrm{~ms})$ used in this simulation.

end boundary conditions. As expected there is no qualitative difference between simulations with the periodic- and fixedend boundary conditions except near the boundaries.

During the chirping phase of the driver many moving ILMs appear which then coalesce into several trapped ILMs. Most of the ILMs appear to die out after only a few hundred periods of oscillation. In this particular simulation, four modes can be identified that persist much longer than the others. These are identified in Fig. 11(b) as LS1, LS2, LS3, and NL1. The first three of these are trapped at particular lattice sites and in addition, have their frequency locked to that of the driver. Because of this synchronization with the driver there is a continual transfer of energy to the ILMs, which keeps them pinned at the particular lattice sites and prevents them from decaying. The fourth large-amplitude ILM, NL1, fails to frequency lock to the driver in the $\mathrm{cw}$ region, and hence ultimately decays after a time lapse of $\sim 2600$ periods. Once the driver has been turned off, the three locked states decay. Mode LS3 is seen to decay peacefully, maintaining its symmetry without further motion in the lattice. In contrast, LS1 and LS2 are close enough together so that once the decay begins and they become unpinned, they repel one another, and move in the lattice.
Figure 11(c) shows the average energy for a particular locked ILM (LS3) as a function of time as determined by

$$
E_{L S 3}=\sum_{j=43}^{45} E_{j},
$$

where the $E_{j}$ are defined by Eq. (4). The low frequency oscillation of the average mode energy at frequency $0.0056 f_{a}$ decays slowly over a long time interval. It requires the presence of the driver and disappears immediately after the driver is turned off. The figure shows that during the chirp, the energy of this mode increases with time to an amplitude value compatible with the fixed synchronization frequency of the driver. Thus, the amplitude of such a locked ILM is rigidly connected to the driver frequency.

To explore the development of locked ILMs from another view, the double Fourier transform of the cantilever motion over a specific time interval is taken. The resulting time development of excitations both in real space and in $k$ space is shown in Fig. 12. The energy density as a function of time for this particular simulation is presented in Fig. 12(a). The same parameters are used as those for Fig. 11; only the initial noise is different. The frequency chirp ( $f_{a}$ $\rightarrow 1.027 f_{a}$ ) lasts for 2500 periods [left solid vertical line in (a)]. At long times only one locked ILM appears. An examination of the energy versus time for the center cantilever and two neighboring sites shows a monochromatic strength oscillation frequency at $0.0056 f_{a}$ the same as was described above for LS3. The pulse is turned off at time $=7500$ periods [right solid vertical line in (a)]. By time-space Fourier transforming the displacement over the different time intervals identified by the dotted lines: $b, c, d, e, f$, and $g$, in Fig. $12(\mathrm{a})$, the $(\omega, k)$ excitation plots shown in frames $(\mathrm{b})-(\mathrm{g})$ are obtained. The dotted curves in these six frames identify the linear dispersion curve of the optic branch for the di-element cantilever array. Figures 12(b) and 12(c) show time cuts during the chirp. The position of the nonlinear dispersion curve and the interference between different ILMs can be seen in Fig. 12(c). The early and late stages of the single locked state appear in Figs. 12(d) and 12(e). Figures 12(f) and 12(g) identify the decaying state. A brief description of these findings has been presented earlier. ${ }^{68}$

The time evolution of Fig. 12 shows that initially the region above the linear dispersion curve is almost uniformly excited [Fig. 12(b)] in $k$ space, while in real space there are many different levels of excitations and localized excitation speeds. By the middle of the chirp, several horizontal-line tracks, characteristic of stationary ILMs, emerge in the $k$-space representation [Fig. 12(c)]. In the initial stage after the chirp one strong horizontal excitation is accompanied by flat, relatively weak side bands as shown in Fig. 12(d). The side bands are caused by the strength modulation of the locked ILM. Both the background and these side bands to the locked mode are nearly gone by Fig. 12(e). The blue line at the $151 \mathrm{kHz}, k=0$ point, in Fig. 12(e), is a real feature which occurs at the driver frequency. It comes about because the center of the locked ILM is in phase with the driver, while the rest of the lattice is out of phase. In $k$ space, the locked ILM is represented by a near uniform distribution while the 

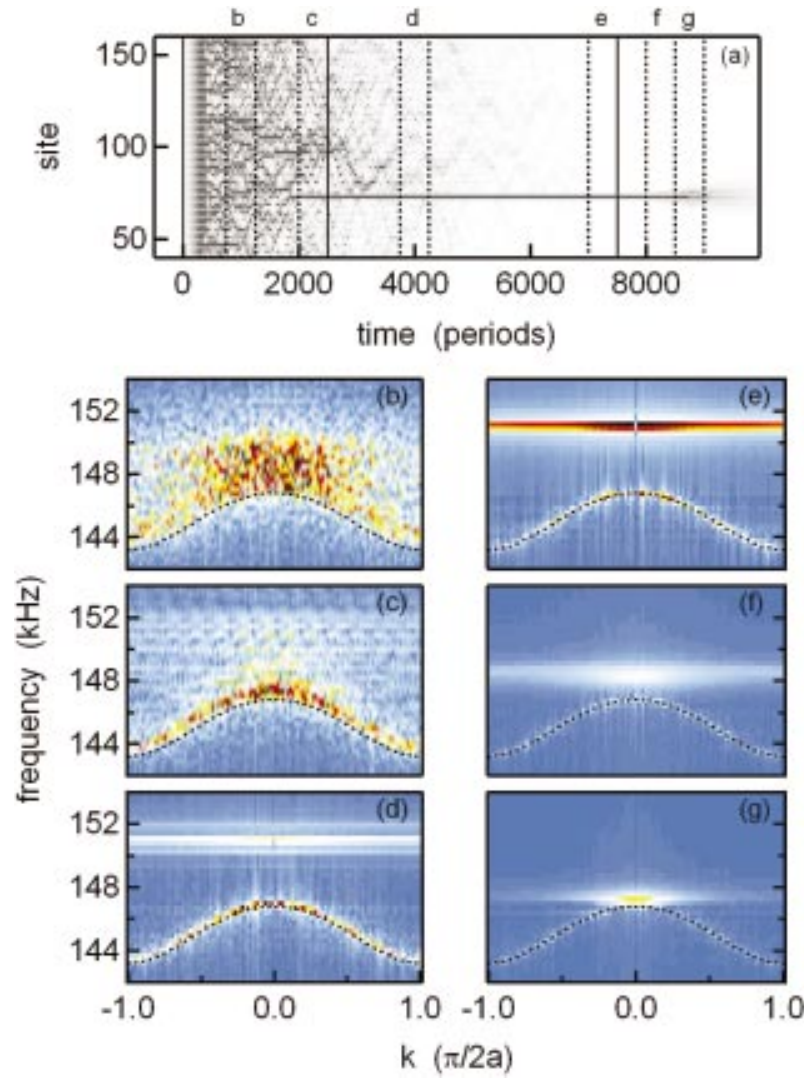

FIG. 12. (Color) Simulated formation, locking and decay of an ILM in the $k$ space. (a) Energy density as a function of time. All parameters used are the same as for Fig. 11, except for the initial noise. The pulse is turned on at time $=0$ and off at 7500 periods. Chirping starts from the top of the band $f_{a}$, and ends at $1.027 f_{a}$ at time $=2500$ periods. The solid lines show when the chirp ends and when the pulse is turned off. Only one ILM is trapped during the $\mathrm{cw}$ part of the pulse. A low frequency oscillation of $0.0056 f_{a}$ is observed at the center ILM site, similar to that shown in Fig. 11(c). The $Q \sim 15$. (b)-(g): Time-space Fourier transform of the displacement in several time windows. The time windows for these frames are indicated by the letters $b-g$ above frame (a). The time development during the initial chirped excitation are shown in frames (b) and (c) locked state in frames (d) and (e), and the decaying ILM state in frames (f) and (g). Dotted curves in frames $(\mathrm{b})-(\mathrm{g})$ identify the linear dispersion curve for the upper band. Note the side bands in frame (d) and the blue line at $k=0$ in frame (e). After Ref. 68.

out of phase component of the lattice is represented by a near delta function. These two contributions cancel at $k=0$ producing the blue spot. After the pulse is turned off, the ILM loses energy, and its frequency decreases as shown in Figs. $12(\mathrm{f})$ and $12(\mathrm{~g})$.

\section{Discussion}

Due to the lack of dynamic range and linearity of the amplitude measurement only limited experimental information about ILM sizes has been obtained. Even though the sizes of ILMs shown in Figs. 8 and 9 appear broader than in the simulation shown in Fig. 11, this may be a feature of the experiment, since the measured darkness is not proportional to the amplitude of the cantilever due to the nonlinearity in the optical response of the cantilever motion and the electrical response of the CCD.
The general picture from observations and simulations is that a number of moving localized modes are created initially, but that there are only very few modes, which grow in amplitude and survive until the end of the chirp and even fewer survive until the end of the $\mathrm{cw}$ pulse. From our simulations, these ILMs are frequency locked to the driver. In this way sufficient energy can be transferred to maintain their strongly localized state and fixed vibrational amplitude. Other ILMs that fail to lock their frequency to the driver are seen to decay. In theoretical work Rössler and Page ${ }^{34}$ have reported a locked in-phase driven single-peaked ILM with out-of-phase background for a realistic anharmonic potential. Their description is somewhat similar to that observed here in Figs. 12(d) and 12(e).

Simulations demonstrate that the mobility of localized modes decreases with increasing amplitude, ${ }^{37}$ and if the amplitude becomes large enough, the localized mode is trapped at a site. ${ }^{42}$ Although the nonlinear KG lattice does not support a Peierls-Nabarro potential ${ }^{43,44}$ due to the presence of internal degrees of freedom, ${ }^{40,69}$ a pinning potential of some sort still appears to be a valid concept.

The two trapped ILMs shown in Fig. 10 have maximum amplitude at the short cantilever site, as do the locked ILMs in the simulations shown in Fig. 11(b). Thus, the singlepeaked mode is more stable than the double peaked mode, in agreement with the early studies of stationary and moving ILMs in antiferromagnets ${ }^{14}$ which have both anharmonic intrasite and intersite potentials.

\section{B. Interactions}

\section{Experiment}

Various types of interactions have been identified in which a locked/pinned ILM participates. Figure 13 addresses the interaction of a pinned ILM with traveling ILMs. The data, a magnified image of a section of Fig. 9(a), is presented in Fig. 13(b), and a schematic representation of the time evolution identifying important features is given in Fig. 13(a). After the pulse is turned on at time $=0$, the initial excitation stage followed by modulational breakup into several small amplitude localized excitations is marked as region (A) in Fig. 13(a). Compare with the data in Fig. 13(b). At longer time, three main excitation sequences appear as outlined in Fig. 13(a). The chirp interval extends to $16.2 \mathrm{~ms}$. Within the center sequence (region B), hopping of an ILM across the lattice can be observed. The random hopping motion of an otherwise pinned ILM is a consequence of its interaction with traveling ILMs and/or background excitations associated with the other normal modes of the array. Markers C-1 and C-2 identify collisions between travelingILMs and/or other excitations and a strongly pinned ILM. In each case the traveling excitation appears to be reflected from the stationary one.

In addition to pinned ILMs, an underlying normal mode excitation pattern is another characteristic feature. The ovals shown in Fig. 9 mark three of the many regions where fine grained, small amplitude background excitation patterns can be seen, even after the end of the pulse. These excitations are 


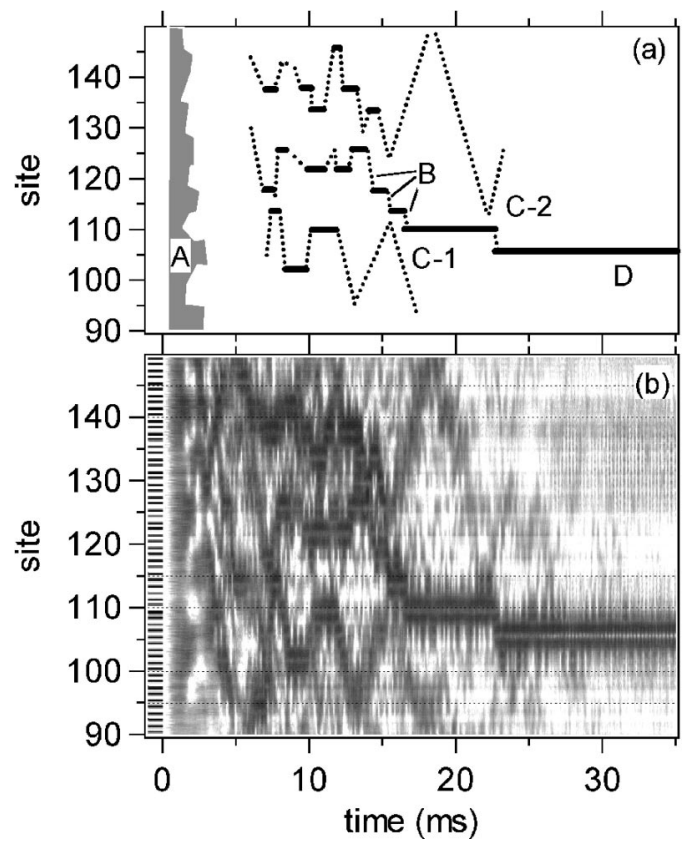

FIG. 13. Experimental demonstration of pinning and hopping of ILMs. Magnified section of part of Fig. 9(a). Enhanced contrast to emphasize only strongly excited modes. Even sites are the shorter cantilever sites. Chirp ends at $16.2 \mathrm{~ms}$. (b) Experimental data: The initial excitation stage (A) is followed by breaking up into several localized excitations, the (B) region shows the hopping of an ILM, region (C-1) and (C-2) illustrate collision and repulsive interaction, and region (D) identifies the final stationary locked state. Note that long-lived strongly excited modes are only at even (short) cantilever sites. The bright center of these ILMs is an experimental artifact.

maintained by the strong driver via nonlinear effects. The exact mechanism of this normal mode excitation is still not completely under experimental control.

The fact that an identical experimental starting condition in Figs. 9(a) and 9(b) give different end results demonstrates the importance of the underlying excitation spectrum of the array. Even when some level of impurities is present (unavoidable for a fabricated system), random ILM creation is possible if the normal mode excitation effect is larger than the impurity effect, which is the case in these experiments. Also the observation of random final conditions during the cw driver interval is experimental evidence that the intrinsic pinning effect dominates any underlying impurity effects. Hence the nonrepeatability of the pinning location strongly supports our claim that the localized excitations in the cantilever array are intrinsic localized modes, as opposed to impurity modes.

\section{Simulations}

Numerical studies show behavior similar to experiment regarding the interaction of a pinned ILM with traveling ILMs. Examination of Fig. 11(b) in the vicinity of the pinned ILMs LS2 and LS3 in the time interval 2000-4000 periods, shows the reflection of localized wavepackets. The reflection of a moving ILM wave packet from a locked ILM comes about because of the effective rigidity of the latter. The amplitude and frequency of a locked ILM are fixed by the frequency and strength of the driver. Since the amplitude of the

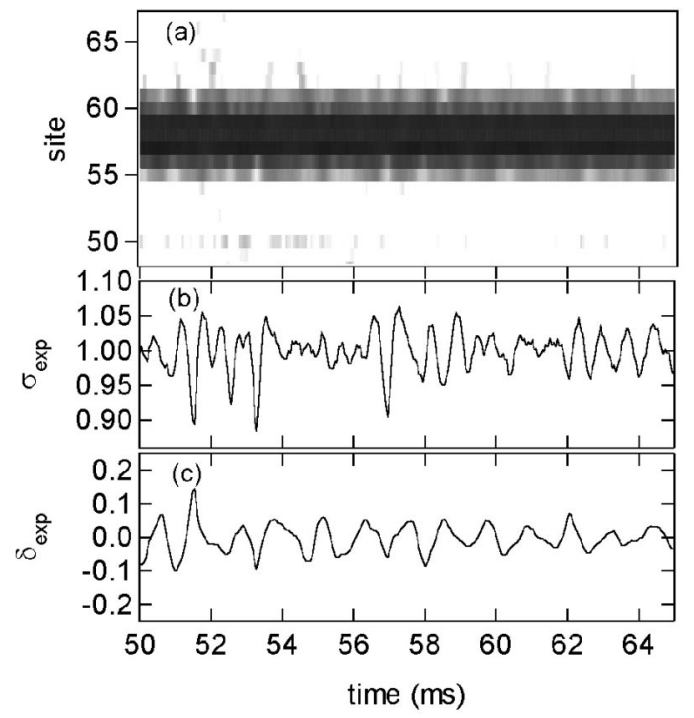

FIG. 14. Low frequency excitations of a pinned ILM. (a) Magnified image of Fig. 8(a). Only cantilever sites shown; the darker the image, the stronger the signal. There is one stable, pinned ILM centered at site 58. (b) The total normalized signal $\sigma_{\text {exp }}$ obtained from the signals at site 55 through site 61 as described in the text. (c) Normalized lateral difference signal $\delta_{\exp }$, as described in the text. The signal at site 58 is almost saturated and cannot be used in the analysis.

locked ILM is fixed and there is no remaining freedom, colliding ILMs are reflected, as though from a boundary.

Most ILMs created during the initial breakup stage of the uniform mode do not lock to the driver, instead they decompose into small amplitude traveling ILM/wavepackets which continually collide with locked ILMs. Near the middle of the chirping stage some locked ILMs are still easily moved by collisions with such traveling modes, since the pinning effect is still weak. Due to the stability difference between the single- and double-peaked modes, ILMs remain longer as single-peaked modes, and when the pinning effect is weak, an ILM appears to hop among its preferred sites as shown by the oval near LS1 in Fig. 11(b). Collisions of an unlocked ILM with small amplitude traveling ILM/wave packets can also be seen in the oval associated with NL1. The hopping process is evident.

The repulsive interaction between two strong ILMs can be seen in Fig. 11(b) to start at around 9000 periods. After the pulse is turned off at 7500 periods, the two ILMs, LS1, and LS2 lose energy, broaden and unpin. Once they are mobile the repulsion between them can be observed.

\section{Excitation of a pinned ILM}

\section{Experiment}

Since a locked ILM appears to be a stable, somewhat rigid excitation state with a fixed amplitude, there is value in examining its time dependence at higher resolution. Figure 14(a) shows an expanded image of the pinned ILM from Fig. 8 (a). Clearly the amplitude near the center of the ILM is oscillating with time. Since the experimental signal for the central site, 58, is saturated, only the signal for the perimeter region can be used in any analysis. Let the total signal from site 55 to site 61 , excluding 58 , be called 

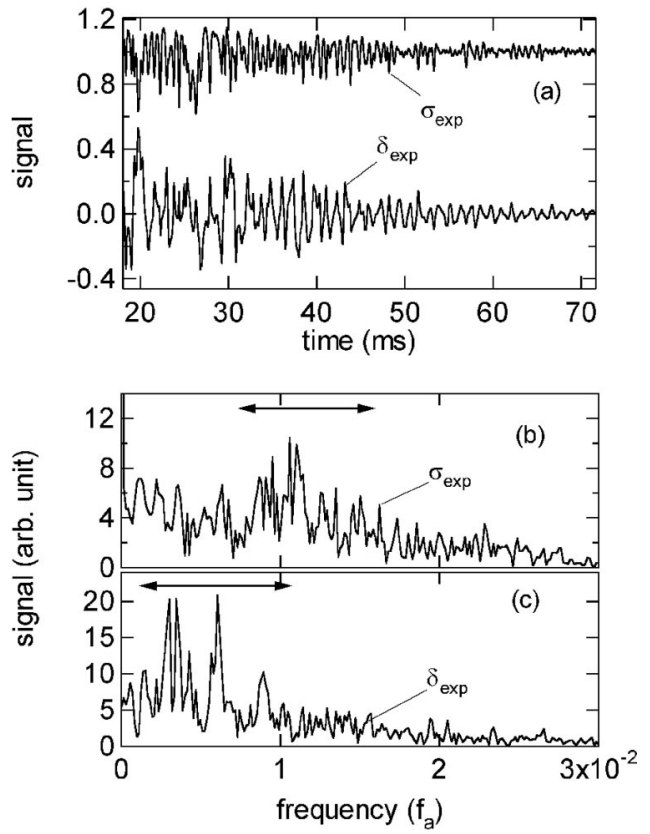

FIG. 15. (a) Transient response of the low frequency excitations of the pinned ILM. The complete time interval of the pinned ILM is shown. (b) The Fourier transform spectrum of the low frequency excitations of the $\sigma_{\text {exp }}$ type. A broad spectrum centered near $0.011 f_{a}$ is shown by the arrow. (c) The Fourier transform spectrum of the low frequency excitations of the $\delta_{\text {exp }}$ type. Several peaks at low frequency region around $0.001 \rightarrow 0.009 f_{a}$ indicated by the arrow are observed.

$$
S=\sum_{\substack{n=55 \\ n \neq 58}}^{61} S_{n},
$$

where $S_{n}$ is the signal at the $n$th site. With the time average of Eq. (6) represented by $\langle S\rangle$, then the dimensionless quantity of interest becomes

$$
\sigma_{\text {exp }}=S /\langle S\rangle \text {. }
$$

The lateral difference signal can be defined in a similar manner. Let

$$
A=\sum_{n=55}^{57} S_{n}-\sum_{n=59}^{61} S_{n}
$$

then

$$
\delta_{\exp }=A /\langle S\rangle .
$$

Figures 14(b) and 14(c) show the measured total amplitude, $\sigma_{\exp }$, and the lateral difference, $\delta_{\exp }$, versus time, respectively. An oscillating $\sigma_{\exp }$ indicates that there are low frequency amplitude modes of the ILM. Figure 14(c) shows an oscillation in $\delta_{\exp }$, which identifies slow lateral motion of the pinned ILM relative to its center. Both of these oscillations represent a slow transient response of the pinned ILM over the entire time that the $\mathrm{cw}$ driver is on. The complete time dependence is shown in Fig. 15(a). The relaxation time is very long compared to the linear energy lifetime $(8.75 \mathrm{~ms})$ of a cantilever.

The Fourier transform of the data in Fig. 15(a) gives the frequencies associated with these small oscillations. The $\sigma_{\exp }$

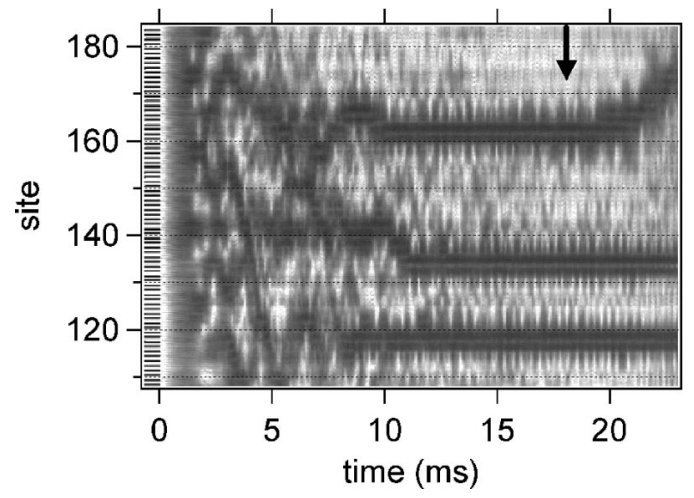

FIG. 16. Another experimental example of small amplitude excitation about a stable ILM state. Magnified section of part of Fig. 9(b). Three ILMs are seen and the lower two become locked. Initially all three show a finegrained oscillation but the top ILM at around time $=15-22 \mathrm{~ms}$, in the region of the arrow, loses energy, the period of the small oscillation increases and finally the ILM becomes unpinned.

spectrum, shown in Fig. 15(b) has one broad peak centered around $0.011 f_{a}$. The $\delta_{\text {exp }}$ spectrum displays several peaks at somewhat lower frequencies.

Another experimental property of these small oscillations is shown in Fig. 16 where the dependence of their frequency versus pinning strength can be seen. This figure is a magnified image of part of Fig. 9(b). The initial traveling localized modes at short times are pinned by the end of the chirp. At longer times there is the fine-grained oscillation pattern that appears on all three stationary localized modes. In some regions these patterns suggest that the center of gravity of the pinned mode is moving side to side while in other regions it appears that the center of gravity is fixed and that the excitation takes a variable intensity character. Note that the period of this oscillation at site 162 (region denoted by the arrow) is larger than for the other two modes shown in the figure. Since the mode at 162 is losing energy and becoming less strongly pinned [see Fig. 9(b) for longer times], the period of this fine structure pattern increases. It is a signature of the pinning strength.

\section{Simulations}

The oscillatory behavior found for the locked ILM state in the experiment provides the motivation to compare the results with simulations. The simulation of the energy versus time for the three central sites of LS3 during the cw region shown in Fig. 11(c) illustrate that a low frequency monochromatic oscillation in the ILM strength is an important feature. A similar strong oscillatory feature in the ILM strength has been observed for the single locked ILM at site 74 shown in Fig. 12(a). Besides this prominent oscillation, which has most of the amplitude on the center site, there are other weaker oscillatory properties of the ILM that can be identified.

A magnified density plot of the energy for an ILM pinned at site 74 in Fig. 12(a) is presented in Fig. 17(a). In this time window where the $\mathrm{cw}$ driver has been on for some time the energy at sites nearby the center of the locked ILM oscillates with time. These time dependent data are now analyzed using similar notation in the same way as the experi- 


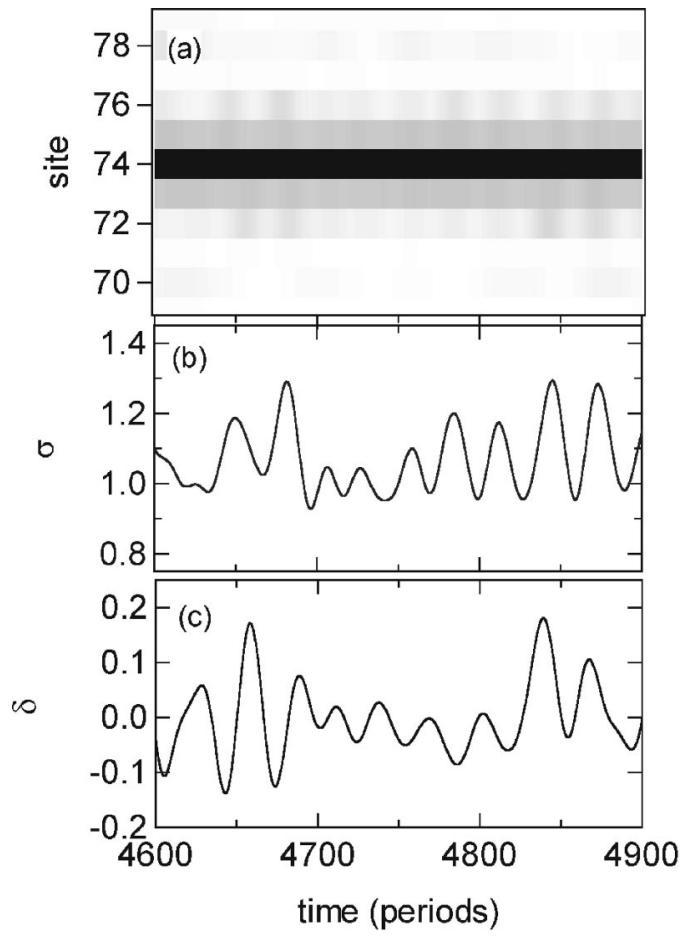

FIG. 17. Simulated demonstration of low frequency excitations about the pinned locked ILM state. (a) Magnified section of Fig. 12(a) which shows one stable ILM pinned at site $=74$. Oscillation of energy between sites 72 and 76 can be seen. (b) Normalized total energy $\sigma$ over sites 72-76 while the cw mode driver is on to bring out low frequency oscillations, as described in the text. (c) Normalized lateral difference energy $\delta$ to bring out other types of low frequency oscillations, as described in the text.

ments were analyzed with Eqs. (7) and (9). The two quantities of interest are (1) the total energy over a restricted region around the pinned mode, excluding the central site, defined as

$$
E=\sum_{\substack{n=72 \\ n \neq 74}}^{76} E_{n},
$$

where $E_{n}$ is the energy at $n$th site. With the time average of Eq. (10) identified as $\langle E\rangle$, the normalized value is

$$
\sigma=E /\langle E\rangle \text {. }
$$

The second quantity (2), the lateral difference energy, is defined as

$$
\Delta=\sum_{n=72}^{73} E_{n}-\sum_{n=75}^{76} E_{n},
$$

to obtain the normalized value

$$
\delta=\Delta /\langle E\rangle .
$$

Equations (11) and (13) now can be used to characterize the small scale oscillatory behavior shown in Fig. 17(a). Figure 17(b) gives the oscillations in the total energy, $\sigma$, the central site, while Fig. 17(c) shows oscillations in $\delta$, indicating lateral motion of the pinned ILM relative to its center.

To identify the significant spectral regions, the Fourier transforms of the two curves in Figs. 17(b) and 17(c) are taken and displayed in Fig. 18. As with the monochromatic low frequency oscillation of the central peak at $0.0056 f_{a}$

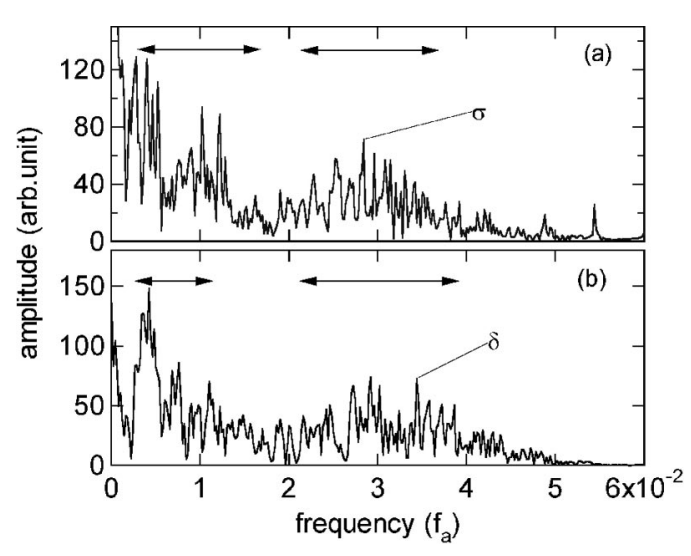

FIG. 18. Fourier transform spectra showing low frequency modes. The $\mathrm{cw}$ driver time interval time $=2500-7500$ periods in Fig. 12 (a) is used. (a) The $\sigma$ spectrum shows response in two different frequency regions, 0 $\rightarrow 0.015 f_{a}$ and $0.02 \rightarrow 0.04 f_{a}$, illustrated by the arrows. (b) The two broad peaks in the $\delta$ spectrum, $0.003 \rightarrow 0.011 f_{a}$ and $0.02 \rightarrow 0.04 f_{a}$ are due to lateral oscillations.

discussed earlier, the characteristic frequencies for the two quantities presented here are very small compared to the highest frequency normal mode, $f_{a}$. There are two broad peaks in the $\sigma$ spectrum, $0 \rightarrow 0.015 f_{a}$ and $0.02 \rightarrow 0.04 f_{a}$ as shown in Fig. 18(a). The lateral mode spectrum $\delta$ also has two broad peaks, $0.003 \rightarrow 0.011 f_{a}$ and $0.02 \rightarrow 0.04 f_{a}$ as shown in Fig. 18(b).

\section{Discussion}

With regard to the stability and rigidity of the locked ILM, several types of deformations from that stable shape have been observed experimentally. One broad frequency region of response is seen for each of the excitation types, namely, $\sigma_{\text {exp }}$ and $\delta_{\text {exp }}$. Since the experimental signal for the central site of such an ILM is saturated, the identities for the different kinds of modes cannot be made as yet. Using the same kind of analysis in the simulations, two broad frequency responses are found for the two kinds of dynamical signatures. In addition a single large amplitude, low frequency mode is found for the central cantilever of the ILM, which appears to represent oscillations in the ILM strength. Given the basic model used to describe the experimental system and the absence of experimental data for the central cantilever, the fact that the small oscillation spectra do not match in detail is perhaps not too surprising. Still a key result has been obtained: both experiment and simulations show that low frequency oscillations are a natural feature of locked ILMs. The strength oscillation found in simulations indicates that the central cantilever response of the ILM must be measured in experiment in order to develop a more complete understanding of these low frequency oscillatory modes and to determine whether or not a more detailed simulation model is warranted.

\section{Trapped ILM oscillations}

\section{Experiment}

After the finish of the cw pulse, oscillatory features can still be detected. The excitation region around site 80 in Fig. 8(b) shows interesting behavior as the pinned ILM decays. 


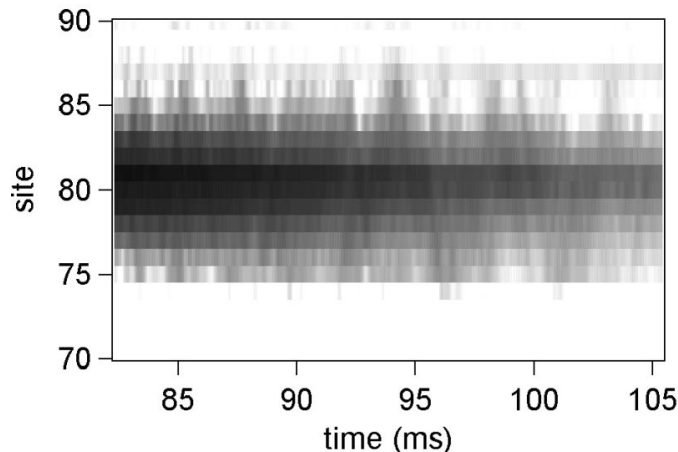

FIG. 19. Trapped excitations oscillating around a pinned ILM. The data are from a section of Fig. 8(a). Only cantilever sites are shown and the darker the image, the stronger the signal. The pulse is turned off at time $=72 \mathrm{~ms}$. Decay of the pinned ILM is shown. From time $=92 \mathrm{~ms}$, traverse patterns due to running wave packets trapped in the ILM envelope appear as oblique lines.

Figure 19 can be characterized as an oscillating wavepacket trapped by the pinned ILM. Only pixels corresponding to cantilever sites are shown in this figure in order to enhance the excitation pattern. The main feature is the pinned ILM, which has evolved from a previously locked ILM at the end of the pulse. As time passes, the amplitude of the center region decreases, and the ILM broadens. Additionally, several running wavepackets trapped within the center region can be identified.

An example of another kind of trapped oscillation about a pinned ILM is shown in Fig. 20. Figure 20(a) presents the magnified image of a portion of Fig. 9(a), which shows a stationary ILM just after the end of the pulse. The bright center in Fig. 20(a) is an optical artifact. The change in the

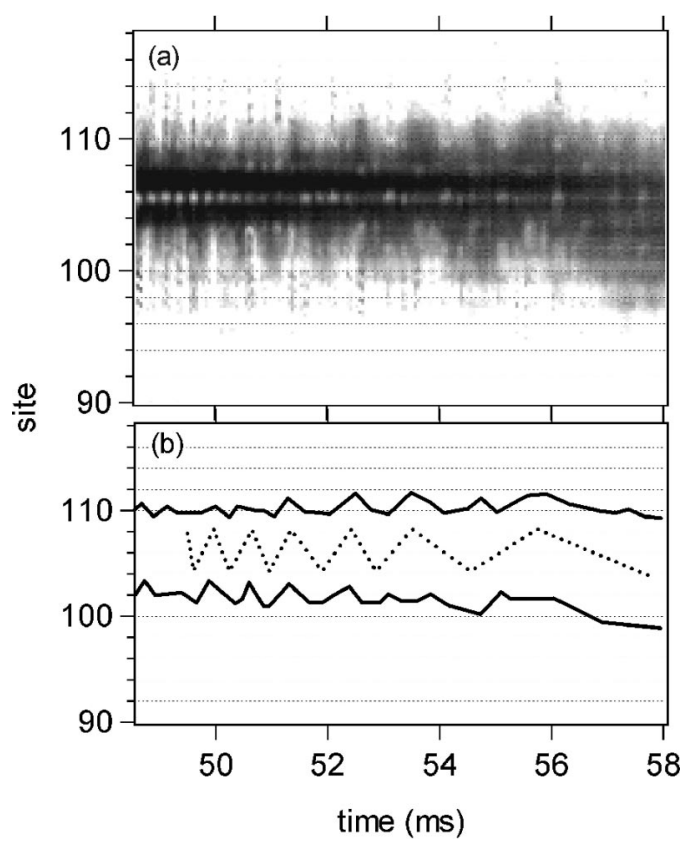

FIG. 20. A trapped excitation oscillating around a pinned but decaying ILM. (a) Experimental image. The ILM becomes unpinned at time $\sim 56 \mathrm{~ms}$ during which time the period of the lateral oscillation increases. (b) Schematic image. This ILM starts to oscillate laterally from time $\sim 50 \mathrm{~ms}$, which is shown as the dotted line.

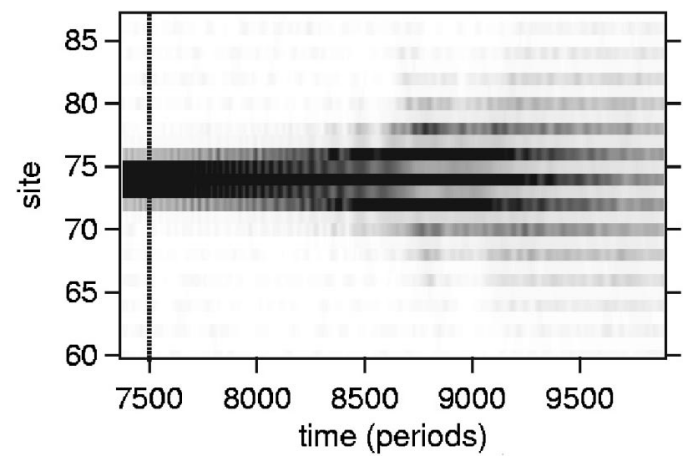

FIG. 21. Simulation of trapped modes in a decaying but pinned ILM. This is a magnified section of Fig. 12(a). The pulse is turned off at time $=7500$ periods. The decay of the pinned ILM is evident from its broadening. After 8700 periods, running wave packets trapped in the ILM envelope appear as oblique patterns.

darkness of the center region with time indicates that the amplitude of the pinned mode is decreasing. Superimposed on this pinned excitation is an oscillating mode. Its period of oscillation increases with time, as shown schematically in Fig. 20(b).

\section{Simulations}

Figure 21 shows the evolution of ILM-trapped wave packets in simulation. This is a magnified picture of a region of Fig. 12(a), after the end of the pulse (dotted line). Initially a variable intensity mode pattern can be seen. Later, a running wavepacket pattern becomes prominent.

Since there is no driver, all the energy for these trapped modes comes from the locked state before the pulse is turned off. Part of this locked mode is converted into the trapped running wave packets, still confined to the original spatial region of the ILM. When the amplitude in the central region becomes sufficiently small so that the pinning effect weakens, and if enough energy is converted to the trapped running modes, then the pinned mode can move. Thus, the trapped running modes and the lateral oscillation of the perimeter are the precursors to the ILM launching from the pinned location.

\section{CONCLUSIONS}

Experiments have been carried out in which the creation, interaction, and relaxation of intrinsic localized modes produced in di-element cantilever arrays have been imaged and recorded. The experimental method permits examination of the vibration envelope of a micromechanical oscillator array. By using this experimental setup together with a chirped driver, the evolution of the unstable uniform mode into ILMs has been examined in some detail. Initially traveling ILMs are created but finally locked ILMs are pinned at specific sites in the array. The ILM hopping motion and subsequent stationary behavior can be explained by an amplitude dependent pinning effect plus the collision with traveling ILMs/ wave packets which stems both from unlocked ILM excitations and normal-mode excitations created by the strong driver. A repulsive interaction between a stable, locked ILM and a moving unlocked ILM has been observed. Small am- 
plitude excitations of the pinned ILM from the stable locked state, and trapped excitations by a pinned ILM also have been identified. The stability and rigidity of locked ILMs depend on the existence of the $\mathrm{cw}$ driver plus damping. By modeling the array with a nonlinear Klein-Gordon lattice, many of the experimental observations can be identified and examined in more detail, such as the synchronization and pinning, the rigidity and dynamics of the locked ILM mode, and the repulsive interaction.

Three possible extensions to these experiments, which would provide more dynamical information, should be mentioned. (1) The combination of single cantilever optics of Fig. 2 with the experimental setup of Fig. 6 to identify the low frequency modulation of the ILM center. (2) Modulate the laser beam close to the driver frequency to observe the displacement amplitude and phase using the same CCD. The cantilever would again modulate the laser beam, and the slow CCD would then pick up the difference frequency signal as long as the difference is smaller than the speed of the camera. This method could be used to observe both the stationary and traveling locked mode. (3) Another extension would be to use optimal control ${ }^{65}$ in this finite array to excite the individual eigenvector of a specific ILM.

\section{ACKNOWLEDGMENTS}

Conversations with J. P. Sethna, R. H. Silsbee, and J. B. Page have been very helpful. Supported by NSF-DMR and CCMR. The fabrication of samples was performed at the Cornell Nanofabrication Facility, which is also supported by the NSF.

${ }^{1}$ A. J. Sievers and S. Takeno, Phys. Rev. Lett. 61, 970 (1988).

${ }^{2}$ J. B. Page, Phys. Rev. B 41, 7835 (1990).

${ }^{3}$ S. A. Kiselev, S. R. Bickham, and A. J. Sievers, Comments Condens. Matter Phys. 17, 135 (1995).

${ }^{4}$ A. J. Sievers and J. B. Page, in Dynamical Properties of Solids: Phonon Physics The Cutting Edge, edited by G. K. Norton and A. A. Maradudin (North-Holland, Amsterdam, 1995), Vol. VII, p. 137.

${ }^{5}$ S. R. Bickham, S. A. Kiselev, and A. J. Sievers, in Spectroscopy and Dynamics of Collective Excitations in Solids, edited by B. Di Bartolo, NATO ASI Series (Plenum, New York, 1997), Vol. 356, p. 247.

${ }^{6}$ S. Flach and C. R. Willis, Phys. Rep. 295, 181 (1998).

${ }^{7}$ A. Franchini, V. Bortolani, and R. F. Wallis, J. Phys.: Condens. Matter 12, 5629 (2000).

${ }^{8}$ R. F. Wallis, A. Franchini, and V. Bortolani, Phys. Rev. B 50, 9851 (1994).

${ }^{9}$ R. Lai, S. A. Kiselev, and A. J. Sievers, Phys. Rev. B 54, R12665 (1996).

${ }^{10}$ S. Rakhmanova and D. L. Mills, Phys. Rev. B 54, 9225 (1996).

${ }^{11}$ R. Lai and A. J. Sievers, Phys. Rev. B 55, R11937 (1997).

${ }^{12}$ S. Rakhmanova and D. L. Mills, Phys. Rev. B 58, 11458 (1998).

${ }^{13}$ R. Lai and A. J. Sievers, Phys. Rev. B 57, 3433 (1998).

${ }^{14}$ R. Lai and A. J. Sievers, Phys. Rep. 314, 147 (1999).

${ }^{15}$ U. T. Schwarz, L. Q. English, and A. J. Sievers, Phys. Rev. Lett. 83, 223 (1999).

${ }^{16}$ L. Q. English, M. Sato, and A. J. Sievers, J. Appl. Phys. 89, 6707 (2001).

${ }^{17}$ M. Sato, L. Q. English, B. E. Hubbard, and A. J. Sievers, J. Appl. Phys. 91, 8676 (2002).

${ }^{18}$ W. Z. Wang, A. R. Bishop, J. T. Gammel, and R. N. Silver, Phys. Rev. Lett. 80, 3284 (1998).

${ }^{19}$ H. Fehske, G. Wellein, H. Buttner, A. R. Bishop, and M. I. Salkola, Physica B 281, 673 (2000).

${ }^{20}$ P. Maniadis, G. P. Tsironis, A. R. Bishop, and A. V. Zolotaryuk, Phys. Rev. E 60, 7618 (1999).
${ }^{21}$ J. L. Ting and M. Peyrard, Phys. Rev. E 53, 1011 (1996).

${ }^{22}$ M. Peyrard, Europhys. Lett. 44, 271 (1998).

${ }^{23}$ N. Theodorakopoulos and M. Peyrard, Phys. Rev. Lett. 83, 2293 (1999).

${ }^{24}$ O. M. Braun, T. Dauxois, and M. Peyrard, Phys. Rev. B 56, 4987 (1997).

${ }^{25}$ J. E. Hammerberg, B. L. Holian, J. Roder, A. R. Bishop, and S. J. Zhou, Physica D 123, 330 (1998).

${ }^{26}$ E. Trias, J. J. Mazo, and T. P. Orlando, Phys. Rev. Lett. 84, 741 (2000).

${ }^{27}$ P. Binder, D. Abraimov, A. V. Ustinov, S. Flach, and Y. Zolotaryuk, Phys. Rev. Lett. 84, 745 (2000).

${ }^{28}$ O. Bang and P. D. Miller, Opt. Lett. 21, 1105 (1996).

${ }^{29}$ A. R. McGurn, Phys. Lett. A 251, 322 (1999).

${ }^{30}$ A. R. McGurn, Phys. Rev. B 61, 13235 (2000).

${ }^{31}$ G. Dugan, Phys. Rev. ST Accel. Beams 2, 094401 (1999).

${ }^{32}$ W. Z. Wang, J. T. Gammel, A. R. Bishop, and M. I. Salkola, Phys. Rev. Lett. 76, 3598 (1996).

${ }^{33}$ D. Nevedrov, V. Hizhnayakov, and A. J. Sievers, Physica B 316, 132 (2002).

${ }^{34}$ T. Rössler and J. B. Page, Phys. Lett. A 204, 418 (1995).

${ }^{35}$ T. Rössler and J. B. Page, Phys. Rev. B 62, 11460 (2000).

${ }^{36}$ J. L. Marín, F. Falo, P. J. Martínez, and L. M. Floría, Phys. Rev. E 63, 066603 (2001).

${ }^{37}$ S. R. Bickham, A. J. Sievers, and S. Takeno, Phys. Rev. B 45, 10344 (1992).

${ }^{38}$ K. W. Sandusky, J. B. Page, and K. E. Schmidt, Phys. Rev. B 46, 6161 (1992).

${ }^{39}$ C. Claude, Y. S. Kivshar, O. Kluth, and K. H. Spatschek, Phys. Rev. B 47, 14228 (1993).

${ }^{40}$ S. Flach and C. R. Willis, Phys. Rev. Lett. 72, 1777 (1994).

${ }^{41}$ S. R. Bickham, S. A. Kiselev, and A. J. Sievers, Phys. Rev. B 47, 14206 (1993).

${ }^{42}$ O. Bang and M. Peyrard, Physica D 81, 9 (1995).

${ }^{43}$ Y. S. Kivshar and D. K. Campbell, Phys. Rev. E 48, 3077 (1993).

${ }^{44}$ D. Cai, A. R. Bishop, and N. Grønbech-Jensen, Phys. Rev. Lett. 72, 591 (1994).

${ }^{45}$ R. Bourbonnais and R. Maynard, Phys. Rev. Lett. 64, 1397 (1990).

${ }^{46}$ Y. S. Kivshar, Phys. Lett. A 161, 80 (1991).

${ }^{47}$ S. R. Bickham, A. J. Sievers, and S. Takeno, in Defects in Insulating Materials, edited by O. Kanert and J.-M. Spaeth (World Scientific, Schloss Nordkirchen, Germany, 1993), Vol. 1, p. 531.

${ }^{48}$ Y. S. Kivshar, Phys. Rev. B 47, 11167 (1993).

${ }^{49}$ Y. S. Kivshar, Phys. Lett. A 173, 172 (1993).

${ }^{50}$ S. A. Kiselev, S. R. Bickham, and A. J. Sievers, Phys. Rev. B 50, 9135 (1994).

${ }^{51}$ W. Chen, Phys. Rev. B 49, 15063 (1994).

${ }^{52}$ S. Lou and G. Huang, Mod. Phys. Lett. B 9, 1231 (1995).

${ }^{53}$ P. Binder, D. Abraimov, and A. V. Ustinov, Phys. Rev. E 62, 2858 (2000).

${ }^{54}$ E. Trias, J. J. Mazo, A. Brinkman, and T. P. Orlando, Physica D 156, 98 (2001).

${ }^{55}$ B. I. Swanson, L. A. Brozik, S. P. Love, G. F. Strouse, A. P. Shreve, A. R. Bishop, W.-Z. Wang, and M. I. Salkola, Phys. Rev. Lett. 82, 3288 (1999).

${ }^{56} \mathrm{~K}$. K. Likharov, Dynamics of Josephson Junction Circuits (Gordon and Breach, Philadelphia, PA, 1986).

${ }^{57}$ A. N. Cleland and M. L. Roukes, Appl. Phys. Lett. 69, 2653 (1996).

${ }^{58}$ D. J. Bishop, P. Gammel, and C. R. Giles, Phys. Today 54 (10), 38 (2001).

${ }^{59}$ D. J. Bishop, C. R. Giles, and S. R. Das, Sci. Am. 284, 88 (2001).

${ }^{60}$ M. Zalalutdinov, B. Ilic, D. Czaplewski, A. Zehnder, H. G. Craighead, and J. M. Parpia, Appl. Phys. Lett. 77, 3287 (2000).

${ }^{61}$ S. Evoy, D. W. Carr, L. Sekaric, A. Olkhovets, J. M. Parpia, and H. G. Craighead, J. Appl. Phys. 86, 6072 (1999).

${ }^{62}$ E. Buks and M. L. Roukes, Europhys. Lett. 54, 220 (2001).

${ }^{63}$ M. Zalalutdinov, A. Olkhovets, A. Zehnder, B. Ilic, D. Czaplewski, H. G. Craighead, and J. M. Parpia, Appl. Phys. Lett. 78, 3142 (2001).

${ }^{64}$ M. Peyrard, Physica D 119, 184 (1998).

${ }^{65}$ T. Rössler and J. B. Page, Phys. Rev. Lett. 78, 1287 (1997).

${ }^{66}$ L. D. Landau and E. M. Lifshitz, Theory of Elasticity (Pergamon, Oxford, 1970), p. 117.

${ }^{67}$ T. Dauxois, M. Peyrard, and C. R. Willis, Phys. Rev. E 48, 4768 (1993).

${ }^{68}$ M. Sato, B. E. Hubbard, A. J. Sievers, B. Ilic, D. A. Czaplewski, and H. G. Craighead, Phys. Rev. Lett. (in press).

${ }^{69}$ S. Flach, C. R. Willis, and E. Olbrich, Phys. Rev. E 49, 836 (1994). 